\title{
K-Mean Algorithm for Image Segmentation Using Neutrosophy
}

\author{
Nadeem Akhtar, Nishi Agarwal, Armita Burjwal \\ Department of Computer Engineering, Aligarh Muslim University \\ Aligarh, India \\ \{nadeemaalakhtar,nishi.agarwal1400, aburjwal\}@gmail.com
}

\begin{abstract}
Image Segmentation is a important step in the major applications such as Image Processing, Recognition Tasks, Object Detection, Medical Imaging etc. Method used for image segmentation is responsible for the quality of resultant segments. High quality segmentation requires a method that segments an image into more accurate and relevant results. This paper introduces a new approach for segmenting an image. It combines two learning algorithms, namely the K-means Clustering and Neutrosophic logic, together to obtain efficient results by removing the uncertainty of the pixels. A Neutrosophic domain is defined to characterize an image into three membership sets: Truth, Falsity and Indeterminacy. The Indeterminacy Set is compared against a threshold value. If Indeterminacy is found to be greater than threshold, which means that the pixel may belong to more than one cluster, we change the intensity of the pixel depending upon the truth value. The K-means Clustering algorithm is then employed on modified pixels to obtain hard clusters. Experimental Results verify that the results obtained are more accurate, thereby improves the quality of segmentation.
\end{abstract}

Keywords-Segmentation, Clustering, K-means, Neutrosophic Set.

\section{INTRODUCTION}

"Image Segmentation is the process of partitioning an image into a set of distinct regions that are independent of each other, but homogenous according to some criterion that are color, texture or intensity" [1][6][9-11]. In Image Analysis it plays an important role [8]. Since, all other stages in Image analysis depend upon this step. Major areas where image segmentation is applicable includes "Navigation of robots, filtering of noisy images, Locate objects like roads and forests in satellite images, Face Recognition, Finger print Recognition, Diagnosis, identification, content-based image retrieval, Locate tumors, Measure tissue volumes, target tracking, Computer guided surgery etc ". [5-6][10-11].

There are different kinds of Image Segmentations such as "Pixel Based Segmentation, Edge Based Segmentation, Region based Segmentation, Model Based Segmentation, Colour Image Segmentation, Gray scale Image Segmentation, Text Segmentation and Theory Based Segmentation". Theory Based Segmentation further includes Clustering Techniques and Neural Network-based segmentation [1-2][6][9-10].

On the basis of the following two properties image segmentation algorithms are classified [1][6][10-11]:
1. Discontinuity: In discontinuity, an image is partitioned when a sharp change in the intensity is encountered. Examples of algorithms based on this technique include edge/boundary detection techniques.

2. Similarity/Homogeneity: In similarity, an image is partitioned on the basis of certain predefined criteria which may be color, size etc. Examples of algorithms based on this technique include threshold, region growing, splitting, merging etc.

"Clustering technique is based on the concept of Similarity. Clustering is defined as the partitioning of a data set into subsets or clusters, so that the data in each cluster share some common characteristic be its size, colour or texture etc." [6][10].A clustering method is considered as good if it produces clusters that are of high quality and have common traits within the same cluster i.e. "high intra-class similarity" and that have different traits as compared to the objects of other clusters i.e. "low inter-class similarity"[10][12]. One of the widely known Clustering methods is K-means Clustering [6].

"K-means clustering is an unsupervised clustering algorithm that partitions the pixels into groups/clusters. Here $\mathrm{K}$ is a positive integer number that denotes the number of clusters. Some criterion can be used to determine the value of $\mathrm{K}$ or it can be an input by the user. K-means algorithm is iterative in nature" [10][12]. This method is popular for image segmentation because it is simple to implement, also it is able to cluster huge data points very quickly [6-7][10]. The proposed method uses K-means Clustering Approach along with Neutrosophy.

"Neutrosophy is a branch of philosophy that studies the origin, nature, scope of neutralities, and their interactions with different ideational spectra" [2][3][5][11][13]. "Neutrosophic Set is a generalization of fuzzy sets, especially Intuitionistic Fuzzy Set "[2][3][4]. In Fuzzy Set, each element had a degree of membership. While, in Intuitionistic Fuzzy Set besides the degree of membership, each element also had a degree of nonmembership [4]. The Neutrosophic Set and Intuitionistic Fuzzy Set differs with each other in the sense that the former one includes one more component i.e. Neutral part besides the truth and falsehood components, that's why the name "Neutrosophic" [4].

The notion of the Neutrosophic set can be understood with the help of an example- "A cloud can be considered a 
Neutrosophic set since its borders are uncertain. Each water drop belongs to the cloud with a Neutrosophic probability. Also, around the cloud there are some scattered water drops, about which we cannot conclude whether they belong to cloud or not i.e. they have indeterminacy [4]. Hence, a cloud comes under the category of Neutrosophic set since its elements i.e. water drops have a degree of membership, non-membership and degree of indeterminacy" [4].

While segmenting an image, there are certain pixels which have a high degree of indeterminacy i.e. they may belong to more than one cluster. This effect is undesirable since the clusters obtained in such case are not of good quality. To deal with this problem, a new approach is proposed. In the proposed approach, Neutrosophy is added before K-means Clustering so that the uncertainty of the pixels can be removed and one pixel gets assigned to only one cluster i.e. hard clusters are obtained as a result.

The remaining paper is organized as follows. Section 2 describes the term Neutrosophic Set. Section 3 gives a detailed description of the introduced algorithm. Section 4 discusses the experimental results on number of images and they are compared against existing segmentation method. Finally, the paper concludes in Section 5.

\section{NeUtrosophic SET}

Let $U$ be a universe of discourse and $M$ be its subset [5][11]. Each element, $x$ that belongs to U possess "a degree of membership, indeterminacy, and non-membership in M". An element, $x$ in $M$, having the degree of membership (T), the degree of indeterminacy (I), and the degree of nonmembership (F) is represented by $\mathrm{x}(\mathrm{T}, \mathrm{I}, \mathrm{F})$.

M is termed as Neutrosophic set, and T, I and F are its Neutrosophic components. For e.g. Let $\mathrm{Z}$ be a Neutrosophic set and $x$ be its element having degree of membership 0.4 in $Z$, the degree of indeterminacy 0.1 in $Z$, and the degree of non-membership 0.5 in $Z$ then it is represented as $\mathrm{x}(0.4,0.1,0.5)[4]$.

\section{Proposed Algorithm}

\section{A. Transform input image into Neutrosophic Domain}

"A Neutrosophic image $\mathrm{P}_{\mathrm{NS}}$ is represented by three memberships sets T, I and F. A pixel $P$ in the image is described as $\mathrm{P}(\mathrm{t}, \mathrm{i}, \mathrm{f})$ and belongs to bright pixel set, $\mathrm{W}$ in the following way: it is $\mathrm{t} \%$ true, $\mathrm{i} \%$ indeterminate, and $\mathrm{f} \%$ false as a bright pixel, where $t$ varies in $T, i$ varies in $I$, and $f$ varies in F'[2][5][11][13].

"The Pixel $\mathrm{P}(\mathrm{k}, \mathrm{l})$ in image domain is transformed into the Neutrosophic domain as $\mathrm{P}_{\mathrm{NS}}(\mathrm{k}, 1)=\{\mathrm{T}(\mathrm{k}, \mathrm{l}), \mathrm{I}(\mathrm{k}, 1), \mathrm{F}(\mathrm{k}, 1)\}$ where $\mathrm{T}(\mathrm{k}, 1), \mathrm{I}(\mathrm{k}, \mathrm{l}), \mathrm{F}(\mathrm{k}, \mathrm{l})$ which are defined as'[2][5][11][13]:

$$
\begin{aligned}
& \overline{\mathrm{z}}(\mathrm{k}, \mathrm{l})=\frac{1}{\mathrm{x} * \mathrm{x}} \sum_{\mathrm{a}=\mathrm{k}-\mathrm{x} / 2}^{\mathrm{k}+\mathrm{x} / 2} \sum_{\mathrm{b}=\mathrm{l}-\mathrm{x} / 2}^{\mathrm{l}+\mathrm{x} / 2} \mathrm{z}(\mathrm{a}, \mathrm{b}) \\
& \delta(\mathrm{k}, \mathrm{l})=\operatorname{abs}[z(\mathrm{k}, \mathrm{l})-\overline{\mathrm{z}}(\mathrm{k}, \mathrm{l})] \\
& \mathrm{T}(\mathrm{k}, \mathrm{l})=\frac{\overline{\mathrm{z}}(\mathrm{k}, \mathrm{l})}{\overline{\bar{z}_{\max }}}
\end{aligned}
$$

$$
\begin{aligned}
& \mathrm{I}(\mathrm{k}, \mathrm{l})=\frac{\delta(\mathrm{k}, \mathrm{l})}{\delta_{\max }} \\
& \mathrm{F}(\mathrm{k}, \mathrm{l})=1-\mathrm{T}(\mathrm{k}, \mathrm{l})
\end{aligned}
$$

Where $\mathrm{z}(\mathrm{k}, \mathrm{l})$ is the intensity value of the pixel $(\mathrm{k}, \mathrm{l}), \overline{\mathrm{z}}(\mathrm{k}, \mathrm{l})$ is the local mean value of $z(k, l)$. And, " $\delta(k, l)$ is the absolute value of the difference between intensity $\mathrm{z}(\mathrm{k}, \mathrm{l})$ and its local mean value $\bar{Z}(\mathrm{k}, 1)$ ".

\section{B. Comparing Neutrosophic Image with Threshold}

If we directly fed the input image to the K-Means algorithm, then the clusters obtained are not clear. There is an uncertainty in the assignment of pixels as a pixel may belong to more than one cluster. For such pixels, indeterminacy value is generally greater.

We have set a threshold value, $\mathrm{Z}$ and compare the indeterminacy value (I) of each pixel with that. If a pixel's I value is greater than the threshold, it implies that the pixel may belong to more than one cluster hence we change its intensity on the basis of its truth value. This will now remove uncertainty of pixel i.e. after K-Means clustering it will be assigned to only one cluster. This paper uses the threshold value, $Z=0.85$ and is determined experimentally [5]. The operation is as follows:

$$
\begin{array}{ll}
\bar{T}(k, l)= \begin{cases}T & I<Z \\
\bar{T}_{z} & I \geq Z\end{cases} \\
\bar{T}_{z}(k, l)=\frac{1}{\mathrm{x} * \mathrm{x}} \sum_{\mathrm{a}=\mathrm{k}-\mathrm{x} / 2}^{\mathrm{k}+\mathrm{x} / 2} \sum_{\mathrm{b}=\mathrm{l}-\mathrm{x} / 2}^{\mathrm{l}+\mathrm{x} / 2} \bar{T}(k, l)
\end{array}
$$

Where $\bar{T}(k, l)$ is the new intensity value of the pixel $(\mathrm{k}, 1)$, $\bar{T}_{z}(k, l)$ is the new local mean value of pixel $(\mathrm{k}, 1)$.

\section{Apply K-Mean Algorithm on the Modified Neutrosophic Image}

"The K-Means Algorithm is as follows: At the beginning, the number of clusters is initialized and centroid of each cluster is chosen arbitrarily. The next step is to compute the Euclidean distance of a pixel from each centroid. The centroid from which the distance of the pixel is minimum (i.e. the centroid to which the pixel is closer), the pixel is assigned to that cluster.

This process is repeated for all pixels. When all pixels are assigned to clusters, their centroids are re-computed. The whole procedure is repeated for new centroid values. This process is repeated again and again until there are no more new assignments i.e. the pixels become stable in a single cluster'[6][10][12].

The flow chart of the discussed algorithm is shown in Fig. 1. 


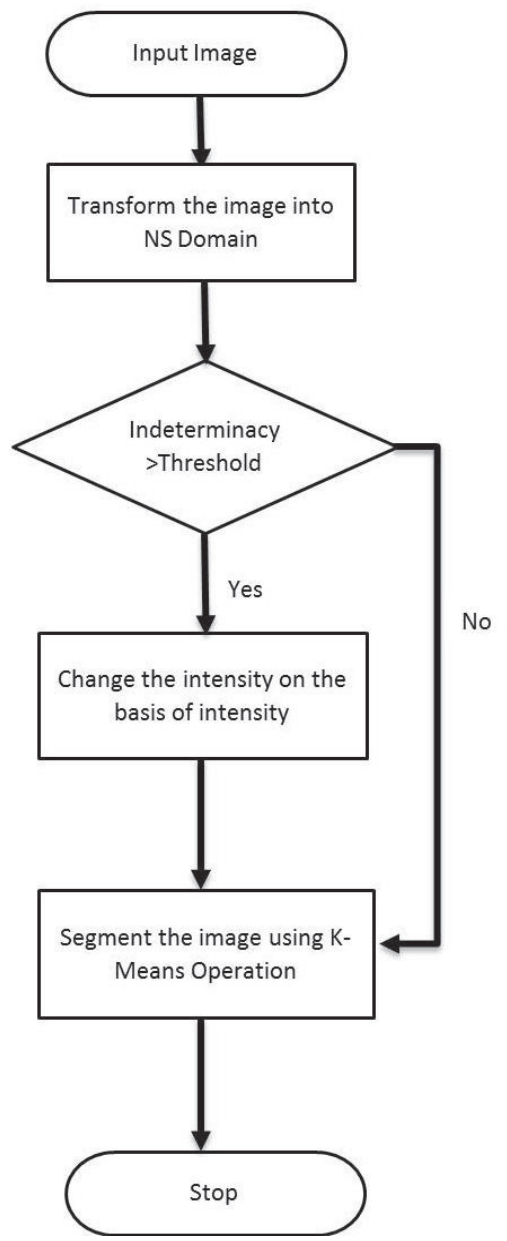

Fig. 1 Flow Chart of Proposed Algorithm

The proposed algorithm is as follows:-

Step1: Convert the image in Neutrosophic Domain;

Step2: Compare each pixel of Neutrosophic Image with threshold and if Indeterminacy is found to be greater than the threshold then modify pixel's intensity using its truth value.

Step3: Initialize number of clusters, say $\mathrm{k}=3$;

Step4: Apply k-means clustering on Modified Neutrosophic Image;

\section{Step5: End;}

\section{EXPERIMENTAL RESULTS}

The proposed algorithm is applied on various images and results are compared with that of conventional K-means Clustering approach. Fig. 2 shows an example of the proposed algorithm. The original image is shown in Fig. 2(a). Fig. 2(b), 2(d) and 2(f) shows the three clusters obtained by the conventional K-Means method while, Fig. 2(c), 2(e) and 2(g) shows the three clusters obtained by the proposed method.

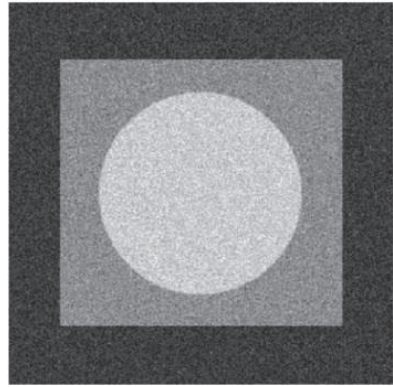

Fig. 2(a)

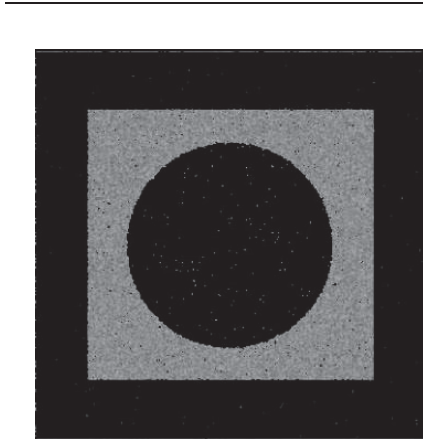

Fig. 2(b)

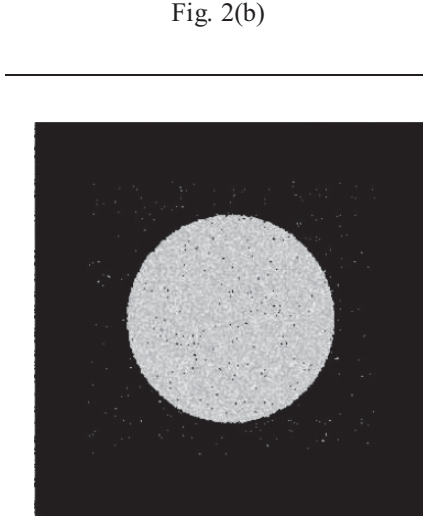

Fig. 2(d)

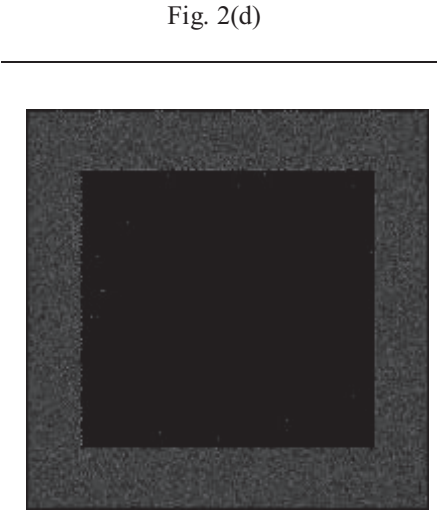

Fig. 2(f)

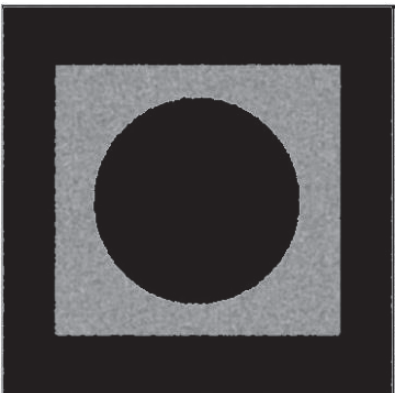

Fig. 2(c)

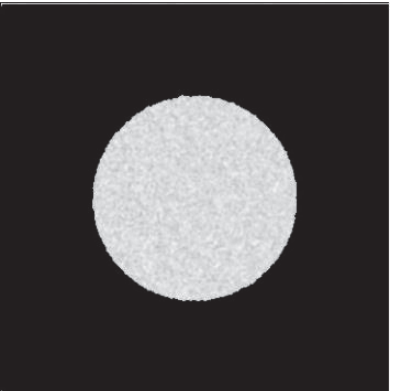

Fig. 2(e)

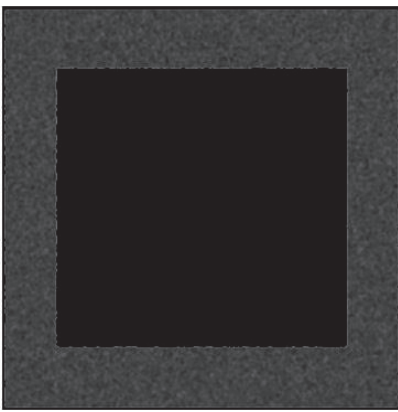

Fig. 2(g)
Fig. 2 an example to illustrate proposed algorithm (a) Original Image (b), (d) and (f) three clusters obtained from K-means Method (c), (e) and (g) three clusters obtained using Proposed Algorithm. 


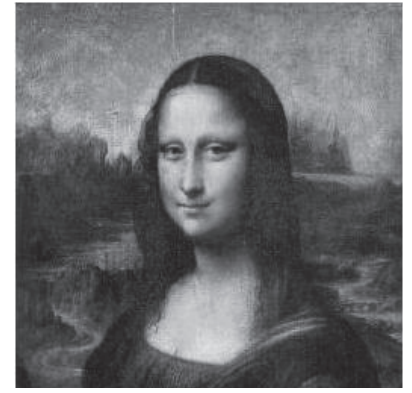

Fig. 3(a)

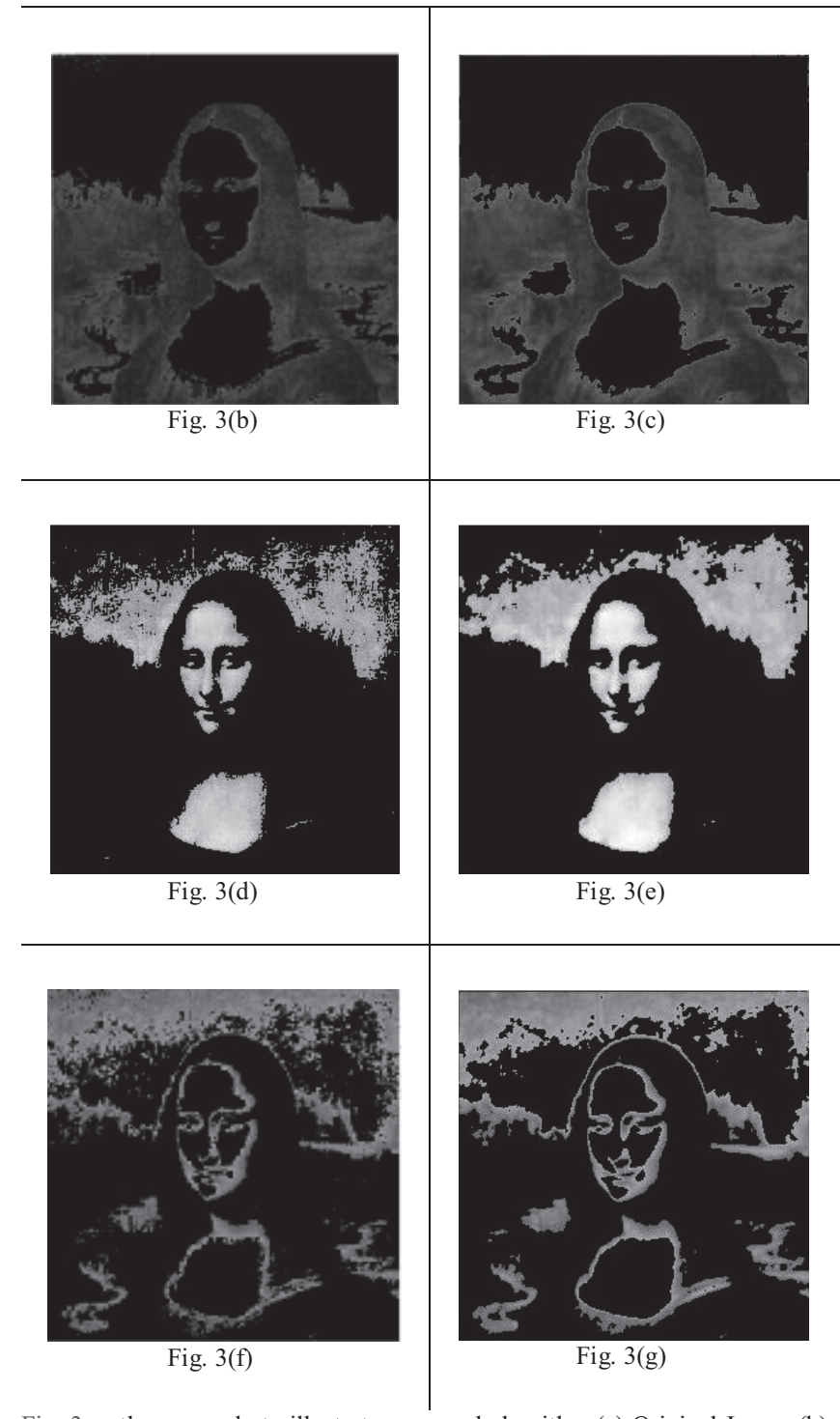

Fig. 3another example to illustrate proposed algorithm (a) Original Image (b), (d) and (f) three clusters obtained from K-means Method (c), (e) and (g) three clusters obtained using Proposed Algorithm.

As it can be seen that, all the three clusters obtained from conventional K-means method contains some part of another clusters along with their own. For example, in Fig 2(b), cluster 1 consists of a rectangle (inner rectangle of original image) as well as some dots (that lies inside the circle in the original image). These dots should not be present in this cluster as they are part of other cluster. Similarly, Cluster 2(Fig. 2(d)) and Cluster 3 (Fig. 2(f)) contain some portion of other clusters. This result is highly undesirable as cluster should contain single object.

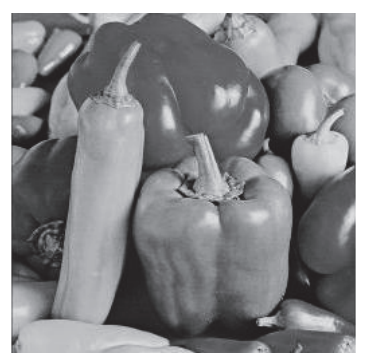

Fig. 4(a)

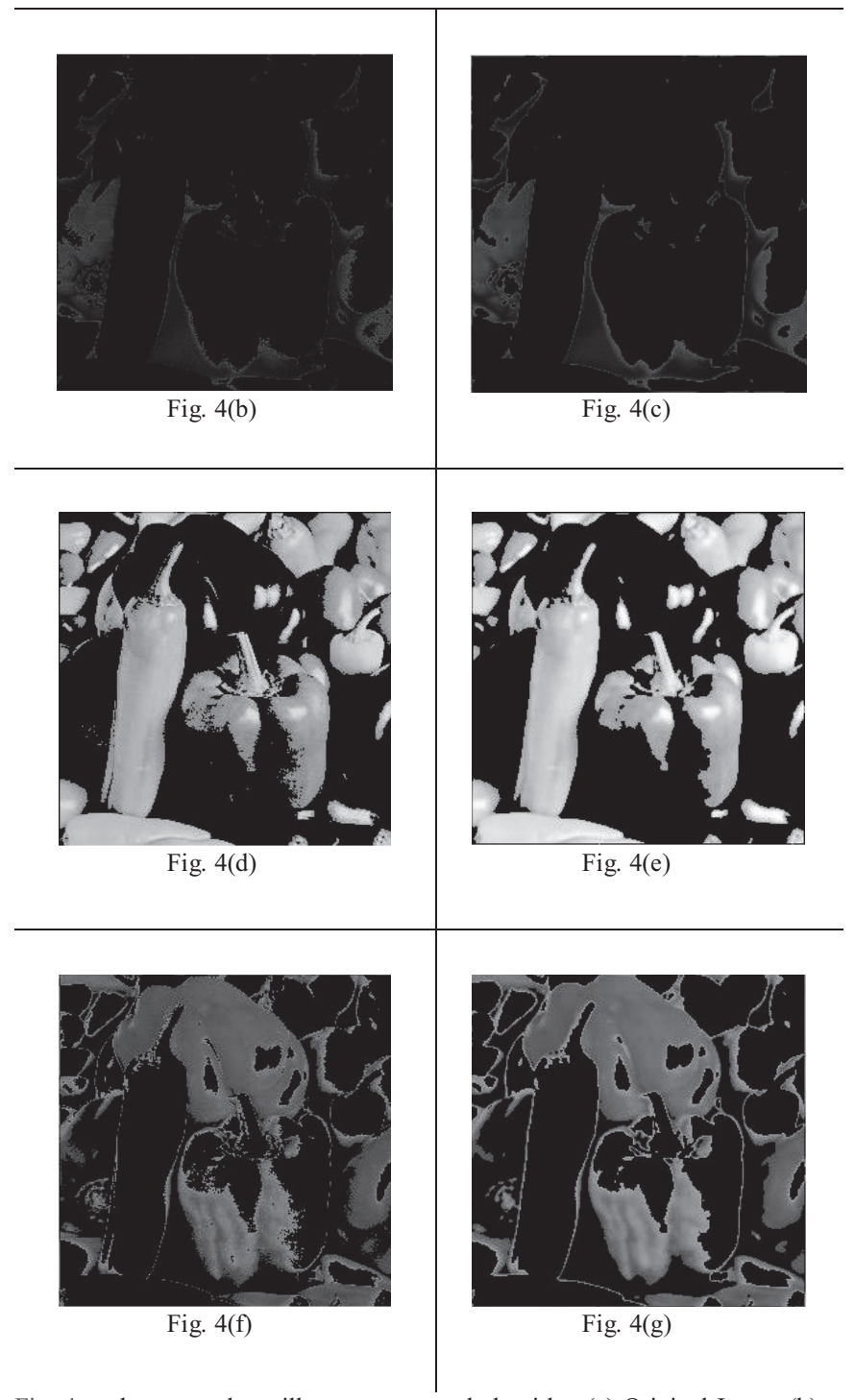

Fig. 4another example to illustrate proposed algorithm (a) Original Image (b), (d) and (f) three clusters obtained from K-means Method (c), (e) and (g) three clusters obtained using Proposed Algorithm. 
But, when the same image is processed using proposed algorithm the results are more accurate. The results of the introduced algorithm are demonstrated in Fig. 2(c), 2(e) and 2(g). These clusters do not contain any extra object. They contain only a single object. Thus, results are improved and the quality of clusters has increased. Fig. 3 and Fig 4 show some other examples of proposed work. Thus, it is clear from these examples that the discussed algorithm gives efficient results in comparison to K-means Clustering algorithm.

\section{CONCLUSION}

Image segmentation is a necessary step in analysis of image. It is important to obtain high quality segments and in that choice of algorithm plays an important role. The clusters obtained from K-means Clustering, are improper as there is a double assignment of pixels. One cluster contains parts of other clusters, which is undesirable. This paper introduces a novel algorithm of Image segmentation, which modifies existing Kmeans clustering using Neutrosophic approach to remove the uncertainty in the assignment of pixels by changing the intensity of the pixel in such a manner that it gets assigned to only one cluster and thus making image suitable for segmentation. A number of experiments were conducted against different images. The quality of clusters and the clusters obtained are fine and smooth as has been proved by the proposed algorithms.

\section{REFERENCES}

[1] Shilpa Kamdi, R.K.Krishna, "Image Segmentation and Region Growing Algorithm", International Journal of Computer Technology and Electronics Engineering, Vol. 2, Issue 1.

[2] Ming Zang, Ling Zhang, H.D. Cheng, "A Neutrosophic approach to Image Segmentation Based On Watershed Method", Signal Processing 90 (2010) 1510-1517.

[3] F. Smarandache, "A Unifying Field in Logics Neutrosophic Logic. Neutrosophy, Neutrosophic Set, Neutrosophic Probability and Statistics", American Research Press, New York, 2003.

[4] Florentine Smarandache, "Neutrosophic Set - A Generalization of the Intuitionistic Fuzzy Set", University of New Mexico, Gallup, NM 87301, USA.

[5] Yanhui Guo, H.D.Cheng, "New neutrosophic approach to image segmentation", Pattern Recognition 42 (2009) 587 - 595.

[6] Jay Acharya, Sohil Gadhiya, KapilRaviya, "Segmentation Techniques For Image Analysis: A Review", International Journal Of Computer Science And Management Research Vol 2 Issue 1 January 2013.

[7] Ali Ridho Barakbah and Yasushi Kiyoki, "A New Approach for Image Segmentation using Pillar-Kmeans Algorithm", International Journal of Information and Communication Engineering 6:2 2010

[8] Hui Zhang, Jason E. Fritts, Sally A. Goldman "Image Segmentation evaluation: A survey of unsupervised methods", Computer Vision and Image Understanding 110 (2008) 260-280.

[9] Krishna Kant Singh, Akansha Singh, "A Study Of Image Segmentation Algorithms For Different Types Of Images", International Journal of Computer Science Issues, Vol. 7, Issue 5,September 2010.

[10] Rajeshwar Dass, Priyanka, Swapna Devi, "Image Segmentation Techniques", International Journal of Electronics \& Communication Technology Vol. 3, Issue 1, Jan. - March 2012

[11] H. D. Cheng, YanhuiGuo And Yingtao Zhang, "A Novel Image Segmentation Approach Based On Neutrosophic Set And Improved Fuzzy C-Means Algorithm", New Mathematics and Natural Computation Vol. 7, No. 1 (2011) 155_171
[12] Ms. ChinkiChandhok, Mrs.SoniChaturvedi, Dr.A.AKhurshid, "An Approach to Image Segmentation using K-means Clustering Algorithm", International Journal of Information Technology (IJIT), Volume - 1, Issue - 1, August 2012.

[13] AbdulkadirSengur, YanhuiGuo, "Color texture image segmentation based on neutrosophic set and wavelet transformation", Computer Vision and Image Understanding 115 (2011) 1134-1144 